\title{
Internationale Zusammenarbeit im Bereich Abfall und Recycling
}

\author{
Bei der Gestaltung der Rohstoffpolitik kommt es \\ darauf an, harmonische Zielbeziehungen zu \\ identifizieren und Zielkonflikte zu berücksichtigen. \\ Die internationale technische Zusammenarbeit \\ im Bereich der Abfall- und Recyclingtechnologie \\ bietet die Chance, sowohl ökologische als auch \\ wirtschafts- und entwicklungspolitische Ziele \\ zu verfolgen. Von Carsten Gandenberger
}

\section{Einleitung}

Auf nationaler Ebene liegen in Deutschland mit der Rohstoffstrategie der Bundesregierung, dem Deutschen Ressourceneffizienzprogramm sowie dem „Entwicklungspolitischen Strategiepapier Extraktive Rohstoffe“ drei rohstoffpolitische Strategien beziehungsweise Programme vor, die jeweils von verschiedenen Bundesministerien federführend formuliert wurden und die in zum Teil sehr unterschiedlichen rohstoffpolitischen Diskursen verankert sind. Hierzu zählt insbesondere der wirtschaftspolitische, der umweltpolitische und der entwicklungspolitische Diskurs über Rohstoffe. Wie angesichts der Vielfalt und Heterogenität der jeweiligen Stakeholder $\mathrm{zu}$ erwarten ist, verfolgt die deutsche Rohstoffpolitik einen breiten Kanon an übergeordneten Zielen, die von den jeweiligen Strategien unterschiedlich akzentuiert werden.

Angesichts der Breite des Zielkanons kommt es bei der Entwicklung rohstoffpolitischer Strategien und der Auswahl geeigneter Politikinstrumente darauf an, harmonische Zielbeziehungen zu identifizieren und mögliche Zielkonflikte angemessen zu berücksichtigen. Eine grobe Übersicht über das Verhältnis der von den drei rohstoffpolitischen Programmen auf nationaler Ebene verfolgten Ziele liefert Abbildung 1.

Wie das Schnittmengenmodell verdeutlicht, gibt es Ziele, die im Schnittmengenbereich aller drei rohstoffpolitischen Politikfelder liegen und die entsprechende Potenziale für die Ent- stehung positiver Synergien bieten, wie zum Beispiel die Verbesserung der Governance im Rohstoffsektor, die Erhöhung der Transparenz von Wertschöpfungsketten und der Aufbau von Rohstoffpartnerschaften. Jedoch kann es trotz vorhandener Synergiepotenziale auch innerhalb der Schnittmenge zu unterschiedlichen Prioritäten und Ausgestaltungsanforderungen kommen, die bei der Suche nach einem kohärenten Ansatz zu beachten sind.

Ziel des vom Umweltbundesamt geförderten und vom Fraunhofer ISI in Zusammenarbeit mit dem Öko-Institut und adelphi bearbeiteten Forschungsprojekts RohPolRess (FKZ 371311) war es dementsprechend, Ansatzpunkte zur Erhöhung der Kohärenz im Bereich der Rohstoffpolitik aufzuzeigen. Hierzu wurden zwölf Kurzanalysen erstellt, in denen verschiedene aktuelle Themen der Rohstoffpolitik analysiert und handlungsnahe Empfehlungen zur Erhöhung der Kohärenz aufgezeigt wurden. Exemplarisch hierfür werden im Folgenden die Ergebnisse der Kurzanalyse Nummer zehn vorgestellt, die den Status quo und die Potenziale der technologischen $\mathrm{Zu}$ sammenarbeit Deutschlands mit Entwicklungs- und Schwellenländern im Bereich der Abfall- und Kreislaufwirtschaft untersucht (Gandenberger 2016).

Unter dem Aspekt der Politikkohärenz bietet ein solcher Ansatz die Möglichkeit, wichtige Ziele der deutschen Umwelt-,

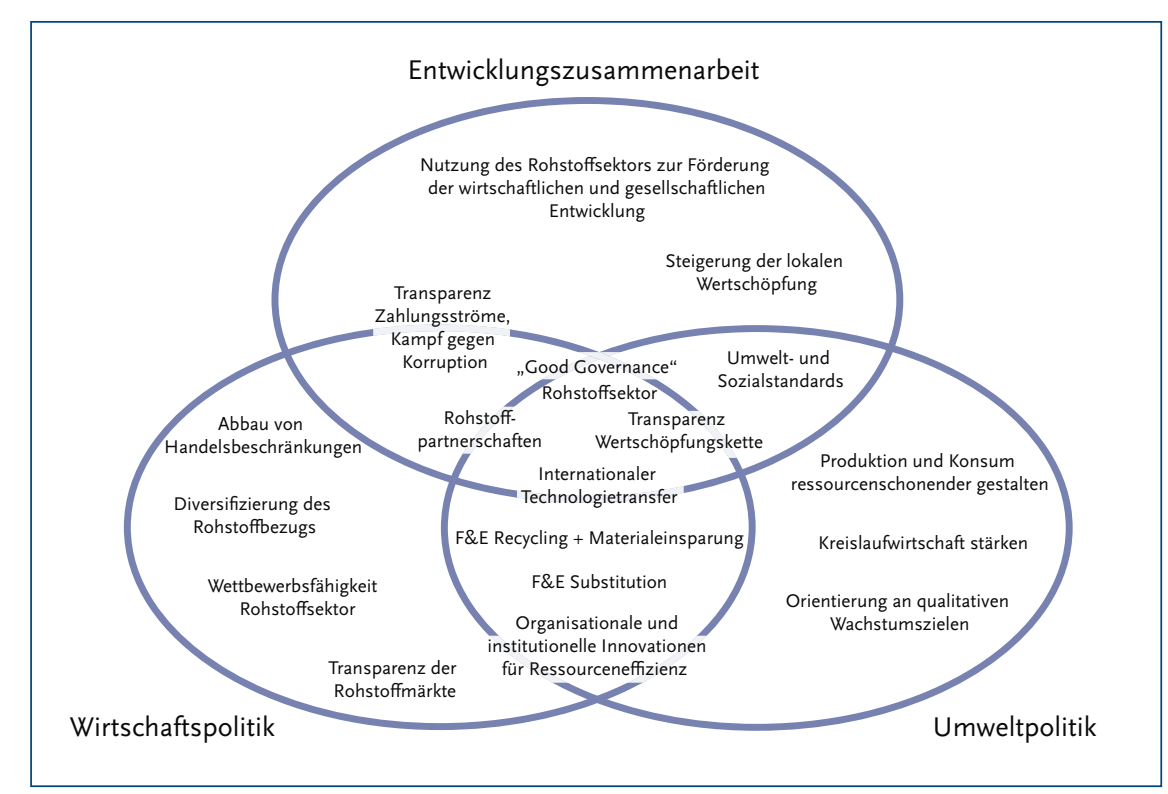

Abbildung 1: Schnittmengenmodell rohstoffpolitischer Ziele

(Quelle: Eigene Darstellung) copying and redistributing the material in any medium or format, provided the original work is properly cited, it is not used for commercial purposes and it is not remixed, transformed or built upon. The access to the digital version of this article is reserved to subscribers of ÖkologischesWirtschaften until two years after the date of publication; after two years it is available to all readers. 
Wirtschafts- und Entwicklungspolitik gleichermaßen zu erreichen. Wenn Schwellen- und Entwicklungsländer beim Aufbau moderner Strukturen in der Kreislaufwirtschaft unterstützt werden, können zum einen Umweltschäden vermieden werden, die durch den Abbau und die Veredelung von Primärrohstoffen sowie unsachgemäße Recyclingprozesse hervorgerufen werden. Zum anderen kann sich das Angebot von Sekundärrohstoffen erhöhen, was zu positiven Auswirkungen auf die globale Rohstoffverfügbarkeit führt. Insbesondere aber kann ein stärkeres Engagement Deutschlands auf diesem Gebiet dazu beitragen, entwicklungspolitische Probleme der Schwellen- und Entwicklungsländer anzugehen, die mit dem stark zunehmenden Rohstoffverbrauch und den damit zusammenhängenden Abfallströmen ausgelöst werden.

Einige Studien haben sich bereits mit der deutschen Exportförderung im Bereich der Abfall- und Recyclingtechnologien und insbesondere der "German ReTech Partnership“ auseinandergesetzt (Range 2014; Beucker et al. 2014; Bethge/Kuhndt 2010) und entsprechende Empfehlungen für die weitere Ausgestaltung, zum Beispiel Verbesserung der Sichtbarkeit, Reduktion des bürokratischen Aufwands, Konsolidierung der zahlreichen Programme, ausgesprochen.

Im Rahmen dieses Beitrags soll dagegen eine übergreifende Perspektive auf den internationalen Technologietransfer und die Rolle Deutschlands eingenommen werden. Die Literatur zum internationalen Technologietransfer weist zum einen darauf hin, dass der Export nur einer von vielen Transferkanälen ist. Zum anderen umfasst der internationale Technologietransfer nicht nur technologische Hardware, sondern auch technologisches Wissen und Know-how. Neben den Exporten sind daher auch ausländische Direktinvestitionen, die Entwicklungszusammenarbeit, Forschungskooperationen, Lizenzgeschäfte und die Migration von Fachkräften zu berücksichtigen (World Bank 2008). Da einige Technologien aus dem Bereich der Abfall- und Kreislaufwirtschaft auch eine hohe Klimarelevanz besitzen, spielt zudem auch der „Clean Development Mechanismus“ (CDM) des Kyoto-Protokolls eine wichtige Rolle als Transferkanal.

Anhand empirischer Daten wird untersucht, wie sich die technologische Zusammenarbeit Deutschlands mit Schwellen- und Entwicklungsländern in den letzten Jahren entwickelt hat und auf welche Länder sich die Zusammenarbeit konzentriert. Hierauf aufbauend werden Maßnahmen diskutiert, die $\mathrm{zu}$ einer Intensivierung der internationalen Zusammenarbeit im Bereich der Abfall- und Kreislaufwirtschaft führen können und die über einen engen Fokus auf die technologische Hardware hinausgehen. Bei der Entwicklung entsprechender Vorschläge ist zu berücksichtigen, dass sich Schwellen- und Entwicklungsländer hinsichtlich vieler relevanter Kontextfaktoren von den Industrieländern unterscheiden. Bei der Formulierung von Handlungsempfehlungen wird hierbei zusätzlich an die wissenschaftlichen Diskussionen über die internationale Diffusion von Klimaschutztechnologien (Ockwell/Mallett 2012) angeknüpft.

\section{Entwicklung des Rohstoffverbrauchs und der Innovationskapazitäten auf globaler Ebene}

Die Entwicklung des Rohstoffverbrauchs ausgewählter Schwellenländer kann anhand des Indikators „Domestic Material Consumption“ (DMC) beziehungsweise „inländischer Materialverbrauch“ nachvollzogen werden. Der DMC erfasst die Gesamtentnahme an direkt verwertetem Material innerhalb einer Volkswirtschaft. Er ist definiert als die jährliche Menge an Rohmaterial, die im Inland entnommen wird, zuzüglich aller physischen Einfuhren und abzüglich aller physischen Ausfuhren. Er bezieht sich auf den tatsächlichen Verbrauch von Agrarrohstoffen, Holz, Baumineralien, Industriemineralien, Metallen und Energierohstoffen (OECD.Stat 2016). Der DMC wird von der Organisation für wirtschaftliche Zusammenarbeit und Entwicklung (OECD) für die Gesamtheit der OECD-Länder, Brasilien, Russland, Indien, China und Südafrika (BRICS-Staaten) und Indonesien, ermittelt und wird daher für den hier angestrebten internationalen Vergleich herangezogen.

Der DMC der OECD-Länder ist im Verlauf der Jahre 19902010 auf hohem Niveau nahezu konstant geblieben. Dagegen hat sich der DMC Chinas im selben Zeitraum mehr als vervierfacht und lag im Jahr 2009 bereits über dem der OECD-Länder in ihrer Gesamtheit. Für Indien und Brasilien zeigt sich im Vergleich zu China ein deutlich langsamerer Anstieg der DMC, wobei Indien seinen Ressourcenverbrauch in diesem Zeitraum allerdings auch mehr als verdoppelt hat und in Brasilien annähernd eine Verdoppelung erreicht wurde. Der DMC-Wert von Indonesien hatte sich zwischen 1990 und 2003 ebenfalls verdoppelt, war danach jedoch wieder rückläufig, sodass insgesamt "nur“ ein Anstieg von knapp 50\% zu verzeichnen war. Die DMC-Werte Russlands und Südafrikas haben sich dagegen annähernd konstant entwickelt. Insgesamt zeigt sich, dass der wirtschaftliche Aufholprozess der Schwellenländer zum Teil gravierende Auswirkungen auf den globalen Ressourcenverbrauch hat.

Den Daten zum Ressourcenverbrauch soll im weiteren Verlauf gegenübergestellt werden, wie sich die Innovationsaktivitäten im Bereich der Abfall- und Kreislaufwirtschaft entwickelt haben. Methodisch orientiert sich das Vorgehen hierbei an der RohPolRess-Kurzanalyse Nummer acht, die sich mit der internationalen Innovationsdynamik bei Ressourceneffizienztechnologien auseinandersetzt (Sartorius/Gandenberger 2016). Da Innovationsaktivitäten nicht direkt messbar sind, müssen Indikatoren genutzt werden, die diese zumindest näherungsweise erfassen. Daher wurden Patente als Forschungs- und Entwicklungs(F \& E)-relevanter, intermediärer Indikator herangezogen, der gleichzeitig als Frühindikator für die zukünftige technische Entwicklung dient. Konkret wurden Patente für technologische Lösungen für die folgenden abfallwirtschaftlichen Aufgaben in die Betrachtung einbezogen: Sammlung und Transport von Abfällen, Recycling, Abfallverbrennung, Rauchgasreinigung, Kompostierung und Deponierung. 
Die empirischen Daten zum DMC und zur technologischen Leistungsfähigkeit können im nächsten Schritt zusammengeführt werden, um zu untersuchen, inwieweit ausgewählte Länder in der Lage sind, die mit einem hohen DMC einhergehenden ökologischen Belastungen durch technologische Innovationen im Bereich der Abfall- und Kreislaufwirtschaft zu bewältigen. In Abbildung 2 sind die logarithmierten absoluten Mittelwerte für den DMC und die Anzahl der Patente der jeweiligen Länder dargestellt, wobei eine klare Zweiteilung zwischen den Schwellenländern im oberen linken Segment der Grafik und den Industrieländern im unteren rechten Segment deutlich wird. Viele Schwellenländer haben bereits einen hohen DMC-Wert erreicht, aber die technologischen Fähigkeiten für einen angemessenen Umgang mit den dadurch entstehenden Umweltproblemen scheinen im Vergleich zu den Industrieländern noch nicht weit genug entwickelt zu sein.

In Anbetracht dieser Ausgangsituation stellt sich die Frage, ob es für die Schwellenländer einen Entwicklungspfad gibt, der mit einem weniger starken Anstieg des DMC und einem beschleunigten Aufbau der benötigten technologischen Kompetenzen einhergeht.

\section{Kanäle des internationalen Transfer von technologischer Hardware und Know-how}

In der Literatur zum internationalen Technologietransfer werden verschiedene Transferkanäle betrachtet (World Bank 2008; Keller 2004). Unter dem Begriff des internationalen Technologietransfers wird in diesem Zusammenhang nicht allein der grenzüberschreitende Transfer technologischer Hardware verstanden, sondern auch der Transfer von technologischem Know-how und Erfahrung. Diese Ausrichtung steht im Einklang mit der Definition des Weltklimarats. Dieser definiert Technologietransfer als „a broad set of processes covering the flows of knowledge, experience and equipment (...) amongst different stakeholders such as governments, private sector entities, financial institutions, NGOs and research/educational institutions (...) (IPCC 2000, S. 3).“ Im Folgenden werden die verschiedenen Transferkanäle kurz vorgestellt:

- Internationaler Handel: Durch den Import von Kapitalgütern oder Halbzeugen, die eine hohe technologische Komplexität aufweisen und für deren Entwicklung beziehungsweise Produktion eine ausgeprägte technologische Kompetenz notwendig sind, können Unternehmen in den Empfängerländern des Technologietransfers die Effizienz ihrer Wertschöpfungsprozesse steigern und somit indirekt von der Innovationsleistung des Herstellers profitieren (Keller 2004).

- Ausländische Direktinvestitionen: Multinationale Unternehmen investieren beim Aufbau neuer Niederlassungen im Ausland in der Regel in neue Anlagen und vermitteln ihr Wissen über effiziente Produktionsmethoden an lokale Mitarbeiter und Zulieferer (Damijan et al. 2003).

- Internationale F \& E-Kooperationen: Sowohl durch den Aufbau von F \& E-Abteilungen privater Unternehmen im Ausland als auch durch internationale Kooperationen von Universitäten und Forschungseinrichtungen kann explizites und implizites Wissen über Landesgrenzen hinweg vermittelt werden (World Bank 2008).

- Vergabe von Produktions- oder Distributionslizenzen an ausländische Unternehmen: Hierbei wird das einem Produkt zugrunde liegende technologische Wissen offengelegt und an den Lizenznehmer transferiert (World Bank 2008).

- Internationale Migration von Branchenexperten und Wissenschaftlern sowie Einbindung in internationale Expertennetzwerke (Altenburg et al. 2008).

- Die Vermittlung technologischen Wissens und die Unterstützung beim Aufbau entsprechender Kompetenzen in Entwicklungsländern ist ein wichtiges Element der Entwicklungszusammenarbeit.

- Ein spezieller Transferkanal, der unter dem Dach des KyotoProtokolls eingerichtet wurde, ist der CDM. Dieser ermöglicht es Akteuren aus Industrieländern, ihre Emissionsver- 
pflichtungen durch die Umsetzung von Klimaschutzprojekten in Entwicklungsländern zu erfüllen (Murphy et al. 2015).

Um den internationalen Technologietransfer im Bereich Abfall- und Kreislaufwirtschaft und die Rolle Deutschlands zu erfassen, liegen Daten zu Exporten, internationalen F \& E-Kooperationen und dem CDM-Mechanismus in der entsprechenden Granularität vor. Da es sich hierbei um unterschiedliche Datenquellen handelt, kommt es zu Unterschieden hinsichtlich der Erfassung einzelner Technologien. Diese Unterschiede werden in Gandenberger (2016) besprochen.

\section{Empirische Ergebnisse}

\subsection{Exporte}

Mit Blick auf die untersuchten Transferkanäle zeigt sich, dass die deutschen Technologieexporte im Bereich der Abfallund Kreislaufwirtschaft in der Periode 2003-2014 von 33,2 auf 79,2 Milliarden US-Dollar gestiegen sind. Einen wesentlichen Anteil an dieser Entwicklung hatten die Exporte in Schwellenländer, die im betrachteten Zeitraum von 8,1 auf 25,2 Milliarden US-Dollar angestiegen sind und die sich somit mehr als verdreifacht haben. Mehr als verdoppelt haben sich die Exporte in den entwickelten westlichen Volkswirtschaften (unter anderem USA, Japan, Australien, EU-Länder), die von 24,2 auf 50,5 Milliarden US-Dollar angewachsen sind. Die Exporte in Entwicklungsländer haben derzeit nur einen Anteil von 0,3\% am Exportvolumen. Der starke Anstieg der Exporte und der wachsende Anteil der Schwellenländer an den Gesamtexporten legen nahe, dass der internationale Technologietransfer zwischen Deutschland und den Schwellenländern deutlich zugenommen hat. Allerdings ist hierbei zu beachten, dass diese Art des an Kapitalgüter gebundenen Technologietransfers zwar in der Lage ist, die Effizienz der Abfall- und Kreislaufwirtschaft in den Empfängerländern zu steigern, aber vermutlich nur einen relativ geringen Beitrag zum Aufbau von technologischem Wissen leistet, da dieses nicht direkt transferiert wird.

\subsection{Internationale F \& E-Kooperationen}

Als Indikator zur Messung des Wissensaustauschs im Rahmen von internationalen F \& E-Kooperationen wurden internationale Ko-Patente und Ko-Publikationen im Bereich der Abfall- und Kreislaufwirtschaft herangezogen. Hierbei kann davon ausgegangen werden, dass im Zuge der wissenschaftlichen Zusammenarbeit sowohl implizites als auch explizites Wissen ausgetauscht wird. Bei der Betrachtung der drei Technologieführer wird deutlich, dass die USA und Deutschland den Anteil der Ko-Patente an den Gesamtpatenten im Betrachtungszeitraum nahezu verdoppelt haben und somit kooperationsfreudiger geworden sind, während Japan relativ konstant einen sehr geringen Anteil an Ko-Patenten aufweist, was jedoch im Einklang mit der allgemeinen Situation des japanischen Innovationssystems steht und somit keine Besonderheit der Abfall- und Kreislaufwirtschaft darstellt (Neuhäusler et al. 2015).
Die Ergebnisse zeigen, dass deutsche Erfinder die meisten Ko-Patente zusammen mit Forschern aus den USA (68), der Schweiz (57) und den Niederlanden (38) angemeldet haben. Neben weiteren europäischen Ländern sind auch die asiatischen Länder Südkorea (29) und China (15) unter den zehn wichtigsten Partnerländern vertreten. Die Ergebnisse der Patentanalyse wurden durch eine Analyse von Ko-Publikationen deutscher Wissenschaftler auf dem Gebiet der Abfall- und Kreislaufwirtschaft ergänzt und bestätigt. Auch hier sind die USA der wichtigste Forschungspartner, gefolgt von den europäischen Ländern.

Die Daten zeigen, dass vermehrt internationale F \& E-Kooperationen durchgeführt werden und dass durch die Kontakte zwischen Wissenschaftlern auch der Technologietransfer intensiviert wird. Für Deutschland ergibt sich der Eindruck, dass - mit Ausnahme Chinas - internationale Technologiekooperationen vor allem mit anderen, ähnlich weit entwickelten Ländern durchgeführt werden, die vermutlich komplementäre Kompetenzen in die Kooperation einbringen können. Bisher sind F \& E-Kooperationen deutscher Wissenschaftler mit Partnern aus Schwellen- und Entwicklungsländern noch selten.

\subsection{Clean Development Mechanism}

Der CDM wurde unter dem Dach der UN-Klimarahmenkonvention als einer der drei sogenannten flexiblen Mechanismen des Kyoto-Protokolls eingerichtet. Durch den CDM können die im Annex 1 des Kyoto-Protokolls aufgeführten Industrieländer Emissionszertifikate erwerben, indem sie in Entwicklungsländern Klimaschutzprojekte umsetzen. Da durch eine angemessene technische Behandlung organischer Abfälle nicht nur klimaschädliche Methanemissionen vermieden, sondern auch Bio- beziehungsweise Deponiegas als alternativer Energieträger gewonnen werden, spielen Projekte aus dem Abfallbereich auch im Kontext des CDM eine bedeutende Rolle. Von gegenwärtig 8.475 in der CDM-Pipeline gelisteten Projekten stammen 1.714 aus den Bereichen „landfill gas" und "methane avoidance“ (UNEP/DTU CDM-Pipeline, Stand 1.7.2016). Die Struktur der Gastländer von CDM-Projekten im Abfallbereich zeigt, dass neben China auch viele andere Schwellenländer CDM-Projekte beheimaten und voraussichtlich von dem damit verbundenen Technologietransfer profitieren. Die Struktur ist damit im Abfallbereich deutlich diversifizierter als im CDM-Sektor insgesamt, der stark auf die Gastländer China und Indien konzentriert ist. Allerdings muss auch bei diesem Transferkanal festgestellt werden, dass die „least developed countries" nur sehr wenige CDM-Projekte beheimaten und somit auch hier kaum vom internationalen Technologietransfer profitieren.

\section{Handlungsempfehlungen}

Um den internationalen Technologietransfer weiter $\mathrm{zu}$ intensivieren, können sowohl aufseiten der Technologiegeberals auch der Technologienehmerländer Maßnahmen ergrif- 
fen werden. Technologiegeberländer wie Deutschland sollten in ihren staatlichen Innovationsförderprogrammen die spezifischen Anforderungen und Bedürfnisse von Schwellen- und Entwicklungsländern verstärkt aufgreifen. In Entwicklungsländern werden häufig einfache, robuste und kostengünstige technologische Lösungen benötigt, die nicht ohne Weiteres mit dem klassischen High-Tech-Innovationsparadigma der Industrieländer kompatibel sind. Vor diesem Hintergrund erscheinen gezielte Forschungsprogramme zur Förderung sogenannter frugaler Innovationen sinnvoll. Neben Anpassungen im Bereich der Technologie, setzt eine erfolgreiche Technologiediffusion in Schwellen- und Entwicklungsländern in der Regel auch Anpassungen des Geschäftsmodells der beteiligten Unternehmen voraus. Bestehende Erfahrungen mit der Diffusion frugaler Innovationen zeigen, dass hierbei das Angebot begleitender Dienstleistungen, innovative Finanzierungsmodelle sowie maßgeschneiderte Informationskampagnen eine wichtige Rolle spielen. Da die am wenigsten entwickelten Länder bisher kaum vom internationalen Technologietransfer profitieren, sollten zielgerichtete Programme entwickelt werden, die gezielte Anreize für private und staatliche Akteure setzen und entsprechende Risiken abfedern. Schließlich sollte die staatliche Entwicklungszusammenarbeit im Bereich der Abfallund Kreislaufwirtschaft und die bestehende Zusammenarbeit in der „German ReTech Partnership“ weiter ausgebaut werden.

In Bezug auf die Empfängerländer wird in der Diskussion um die Beschleunigung des internationalen Transfers von Klimaschutztechnologien darauf verwiesen, dass transparente und zeitlich stabile staatliche Initiativen zur Erhöhung der Nachfrage ein wichtiger Treiber des Technologietransfers sind. Ein weiterer wichtiger Aspekt ist der Abbau von Handels- und Investitionshemmnissen für ausländische Unternehmen, was teilweise aber im Widerspruch zum Bestreben vieler Schwellenländer steht, die eigene industrielle Entwicklung voranzutreiben (Gallagher 2014; Lewis 2013). Für eine Anpassung von Technologien aus dem Ausland sowie die eigenständige Entwicklung von Innovationen müssen die nationalen Innovationssysteme entsprechend gestärkt werden. Für die Klimaschutztechnologien wurde hierzu der Vorschlag gemacht, in Entwicklungsländern spezifische Institutionen einzurichten, die sich dem Aufbau klimarelevanter Innovationssysteme widmen und die entsprechenden Transformationsprozesse koordinieren und vorantreiben (Ockwell/Byrne 2015). Dieser Vorschlag könnte auch auf die Abfall- und Kreislaufwirtschaft übertragen werden.

Ähnlich wie die Klimapolitik hat auch die Rohstoffpolitik eine globale Problemdimension, die eine Intensivierung der internationalen Zusammenarbeit erforderlich macht. Wie die Ausführungen zeigen, ist die internationale technische Zusammenarbeit mit Schwellen- und Entwicklungsländern ein anspruchsvolles, aber bedeutsames strategisches Handlungsfeld der deutschen Rohstoffpolitik, das zukünftig weiter ausgebaut werden sollte.

\section{Literatur}

Altenburg, T./Schmitz, H./Stamm, A. (2008): Breakthrough? China's and India's Transition from Production to Innovation. In: World Development 36/2: 325-344.

Chaminade, C./Lundvall, B.-A./Vang-Lauridsen, J./Joseph, KJ (2009): Designing innovation policies for development: towards a systemic experimentation-based approach. In: Lundvall, B. A. et al. (Hrsg.): Handbook of Innovation Systems and Developing Countries. Cheltenham, Edward Elgar. 360-379.

Damijan, J. P./Knell, M./Majcen, B./Rojec M. (2003): Technology transfer through FDI in top-10 transition countries: How important are direct effects, horizontal and vertical spillovers? In: William Davidson Institute Working Paper No. 549. www.dx.doi.org/10.2139/ssrn.404241

Gallagher, K. S. (2014): The Globalization of Clean Energy Technology: Lessons from China. Cambridge (USA), MIT Press.

Gandenberger, C. (2016): Die technologische Zusammenarbeit Deutschlands mit Entwicklungs- und Schwellenländern im Bereich der Abfall- und Kreislaufwirtschaft - Status quo und Zukunftsperspektiven. Kurzanalyse im Rahmen des Projekts RohPolRess. www.umweltbundesamt.de/ dokument/die-technologische-zusammenarbeit-deutschlands

IPCC (Hrsg.) (2000): Methodological and technological issues in technology transfer: summary for policymakers: A special report of IPCC Working Group III. Intergovernmental Panel on Climate Change. Cambridge (UK), Camridge University Press.

Keller, W. (2004): International Technology Diffusion. In: Journal of Economic Literature, 42/3: 752-782.

Lewis, J. (2013): Green Innovation in China: China's Wind Power Industry and the Global Transition to a Low-Carbon Economy. New York, Columbia University Press.

Murphy, K./Kirkman, G. A./Seres, S./Haites, E. (2015): Technology transfer in the CDM: an updated analysis. In: Climate Policy, 15/1: 1-19.

Nagaoka, S./Motohashi, K./Goto, A. (2010): Patent Statistics as an Innovation Indicator. In: Hall, B. H./Rosenberg, N. (Hrsg.): Handbook of the Economics of Innovation, Volume 2, Amsterdam, Elsevier. 1083-1127.

Neuhäusler, P./Rothengatter, O./Frietsch, R./Feidenheimer, A. (2015): Patent Applications - Structures, Trends and Recent Developments 2014. Fraunhofer Institute for Systems and Innovation Research ISI. Studien zum deutschen Innovationssystem, Nr. 5-2015. Karlsruhe.

Ockwell, D./Byrne, R. (2015): Improving technology transfer through national systems of innovation. Climate relevant innovation-system builders (CRIBs). In: Climate Policy, 16/7: 1-19.

Ockwell, D./Mallett, A. (Hrsg.) (2012): Low-carbon technology transfer. From rhetoric to reality. London, Routledge.

OECD.Stat (2016): Environment Database - Material resources. www.stats.oecd.org/oecdstat_metadata/showmetadata. ashx?dataset=material_resources

Sartorius, C./Gandenberger, C. (2016): Entwicklung der Innovationsdynamik bei Ressourceneffizienztechnologien, Kurzanalyse im Rahmen des Projekts RohPolRess. www.umweltbundesamt.de/dokument/christiansartorius-carsten-gandenberger-fraunhofer

World Bank (Hrsg.) (2008): Global Economic Prospects. Technology Diffusion in the Developing World. Washington, DC.

\section{AUTOR + KONTAKT}

Dr. Carsten Gandenberger ist Wissenschaftler am Fraunhofer-Institut für System- und Innovationsforschung ISI. Seine Forschung konzentriert sich auf die Analyse der Entstehungsbedingungen von Nachhaltigkeitsinnovationen, Umwelt- bzw. Nachhaltigkeitsmanagement und Umweltpolitik.

Fraunhofer-Institut für System- und Innovationsforschung ISI, Breslauer Str. 48, 76139 Karlsruhe. E-Mail: carsten.gandenberger@isi.fraunhofer.de, Website: www.isi.fraunhofer.de

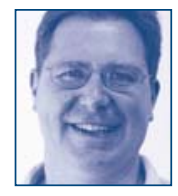

